\title{
A NOVEL TWO STAGE CARRIER FREQUENCY OFFSET ESTIMATION AND COMPENSATION SCHEME IN MULTIPLE INPUT MULTIPLE OUTPUT-ORTHOGONAL FREQUENCY DIVISION MULTIPLEXING SYSTEM USING EXPECTATION AND MAXIMIZATION ITERATION
}

\author{
Malarvezhi Pandy and Kumar Ramamoorthy \\ Department of Electronics and Communication Engineering, \\ Faculty of Engineering and Technology, SRM University, Kattankulathur-603203, Tamil Nadu, India
}

Received 2013-09-15, Revised 2013-09-17; Accepted 2013-09-30

\begin{abstract}
Multiple Input Multiple Output-Orthogonal Frequency Division Multiplexing (MIMO-OFDM) is a promising technique to handle impairments of multipath channel. Alternatively, one of its major drawbacks is drift in carrier frequency, called Carrier Frequency Offset (CFO). Due to CFO, Inter Carrier Interference (ICI) occurs and results in a large performance degradation. The study proposes a novel two stage CFO estimation and compensation technique based on Expectation and Maximization (EM) algorithm with an iterative scheme. Unlike other CFO estimation algorithm, in our proposed CFO estimator a search interval is computed in two stages, hence the estimated offset is more optimal. In the first stage, coarse CFO estimation is done whereas fine estimation is performed in the second stage. This two stage computation results in more accurate and faster convergent estimate. From the simulation results it is proved that the Bit Error Rate (BER) performance of proposed method is more close to ideal Cramer-Rao Lower Bound (CRLB) curve.
\end{abstract}

Keywords: Orthogonal Frequency Division Multiplexing (OFDM), Multiple Input Multiple Output (MIMO), Carrier Frequency Offset (CFO), Expectation and Maximization (EM)

\section{INTRODUCTION}

\subsection{MIMO-OFDM}

Broadband wireless multiple access networks have attracted much attention recently. In the IEEE802.16/WiMAX family of standards, Orthogonal Frequency Division Multiple (OFDM) has been selected as a physical-layer technology (Kaur and Singh, 2012).

OFDM is a modulation technique, which is used in frequency selective fading channels, divides a high data rate bit streams in to a number of lower data rate bit streams over subcarriers transmitted simultaneously through a channel. Each sub stream experiences flat fading in narrowband channel (Mathew et al., 2013).
Subcarriers are orthogonal in time domain, which save bandwidth in frequency domain, resulted in ICI cancelation. Additionally, cyclic prefix as a guard interval with a length, longer than delay spread, that is a copy of last samples, in front of each symbol, avoids ISI therefore OFDM technique is a method to remove the effects of frequency selective fading channels and to enhance the bandwidth efficiency (Oh et al., 2005). OFDM system combined with multiple antennas in both sides of link, resulted in MIMO-OFDM system and higher channel capacity (Torabi et al., 2006). These characteristics of OFDM are achieved when the receiver has exact channel information and the system parameters of transmitter and receiver are perfectly matched. But in real scenario these Technology, SRM University, Kattankulathur-603203, Tamil Nadu, India 
ideal condition does not prevail. Hence results in nonideality like CFO. It is known from (Yue et al., 2007) that $\mathrm{CFO}$ will cause severe Inter Carrier Interference (ICI) in OFDM which degrades the system performance. The impact of CFO on OFDM is estimated in (Tseng and Chung, 2013). A time domain method was proposed in (Chung and Phoong, 2010) for the estimation of CFO in MIMO OFDM system. Zhang and Dai (2010) maximum likelihood method was proposed for CFO estimation.

In the study, we focus on the estimation and compensation of CFO in MIMO-OFDM system. In general, applying the maximum likelihood method for estimation of CFO leads to the problem of high complexity (Zhang and Dai, 2010). Thus we resort to the Expectation-Maximization (EM) algorithm and derive an iterative scheme to overcome this difficulty. In view of computational complexity, we divided the estimation process in to two stages. A coarse CFO estimation is the first stage whereas fine estimation is a second stage. A coarse CFO estimation is performed to evaluate the search range for fine estimation. Then the fine estimation is performed for more accurate result.

\section{SYSTEM DESCRIPTION}

\subsection{MIMO-OFDM System with CFO}

Consider a MIMO-OFDM system consist of $\mathrm{N}_{\mathrm{t}}$ transmit antenna, $\mathrm{N}_{\mathrm{r}}$ receive antenna and subcarriers over channel suffer from a CFO. Input data are modulated by M-ary QPSK mapping in transmitter side. Followed by modulation, each modulated signal vector at time index $n$ is encoded by MIMO encoder. Now the signal vector Equation 1:

$$
x_{j}(n)=\left[X_{j}^{0} X_{j}^{1}, \cdots, X_{j}^{N-1}\right]^{T}, j=1,2, \cdots, N_{t}
$$

where, $x_{j}(n)$ denotes the $n$-th transmitted symbol from the $\mathrm{j}$-th transmit antenna and $\mathrm{X}_{\mathrm{j}}^{\mathrm{N}-1}$ denotes the signal corresponding to the $(\mathrm{N}-1)$-th subcarrier. After Inverse Fast Fourier Transform (IFFT) process, the time domain signal $S_{j}[n]$ can be expressed as Equation 2:

$$
S_{j}(n)=F^{H} \cdot x_{j}(n)
$$

F is N-point IFFT matrix defined as Equation 3:

$$
\mathrm{F}^{\mathrm{H}}=\left[\begin{array}{cccc}
\mathrm{w}_{\mathrm{n}}^{-0.0} & \mathrm{w}_{\mathrm{n}}^{-0.1} & \cdots & \mathrm{w}_{\mathrm{n}}^{-0 .(\mathrm{N}-1)} \\
\mathrm{w}_{\mathrm{n}}^{-1.0} & \mathrm{w}_{\mathrm{n}}^{-1.1} & & \vdots \\
\vdots & & \ddots & \vdots \\
\mathrm{W}_{\mathrm{n}}^{-(\mathrm{N}-1) .0} & \mathrm{w}_{\mathrm{n}}^{-(\mathrm{N}-1) .1} & \cdots & \mathrm{w}_{\mathrm{n}}^{-(\mathrm{N}-1) \cdot(\mathrm{N}-1)}
\end{array}\right]
$$

Where:

$$
\mathrm{w}_{\mathrm{n}}=\frac{1}{\sqrt{\mathrm{N}}} \mathrm{e}^{\frac{\mathrm{j} 2 \pi}{\mathrm{N}}}
$$

A matrix $\mathrm{C}$ is inserted in to transmitted signal which behave like cyclic prefix to avoid the problem of inter block interference. Hence Equation 4:

$$
\mathrm{U}_{\mathrm{j}}(\mathrm{n})=\mathrm{C} \cdot \mathrm{S}_{\mathrm{j}}(\mathrm{n})
$$

where, $\mathrm{C}=\left\lfloor 0_{\mathrm{Ng}_{\mathrm{g}}} \times\left(\mathrm{N}-\mathrm{N}_{\mathrm{g}}\right), \mathrm{I}_{\mathrm{N}_{\mathrm{g}}}: \mathrm{I}_{\mathrm{N}}\right\rfloor$. The notation 0 and I denotes the zero and identity matrix respectively with a subscript of the dimension.

The data transmission unit is carried out in burst mode. Each mode consist of D+1 OFDM blocks. The first is preamble block deals with channel and CFO estimation, whereas the remaining D blocks are data. In the study we focus on preamble block. The following description is with the assumption that the fading processes associated with different antenna pairs are uncorrelated and remain static during each block but vary from one OFDM block to another and that the $\mathrm{CP}$ length is longer than or equal to the maximum channel delay spread. All antenna pairs are assumed to be affected by same CFO.

\subsection{Signal on Receiver Side with CFO}

In receiver side, a superimposition of $\mathrm{L}$ faded signals affected by noise from all $\mathrm{N}$ transmit antennas are received. The signal received by $\mathrm{i}$-th receiver antenna is expressed as Equation 5:

$$
r_{i}(m)=e^{\frac{j 2 \pi \theta m}{N}} \sum_{j=1}^{N_{t}} \sum_{l=0}^{L-1} h_{i, j}(l) x_{j}(m-1)+v_{i}(n)
$$

where, $v_{i}(n)$ is a complex Additive White Gaussian Noise (AWGN) with zero mean and variance. After serial to parallel conversion, cyclic prefix is eliminated by multiplying the $\mathrm{C}$ matrix inverse with received signal vector. Hence Equation 6:

$$
\mathrm{y}_{\mathrm{i}}=\mathrm{C}^{-1} \cdot \mathrm{r}_{\mathrm{i}}(\mathrm{m})
$$

The remaining samples are written in $\mathrm{Nx} 1$ vector. Now Equation 7 and 8:

$$
y_{i}=e^{\frac{j 2 \pi \theta N_{g}}{N}} \sum_{j=1}^{N_{t}} \Gamma(\theta) \cdot D_{j} \cdot h_{i, j}(l)+v_{i}(n)
$$


Where:

$$
\Gamma(\theta)=\operatorname{diag}\{1, \exp (\mathrm{j} 2 \pi \theta / \mathrm{N}), \cdots, \exp (\mathrm{J} 2 \pi \theta(\mathrm{N}-1) / \mathrm{N})\}
$$

is a CFO matrix with normalized frequency offset Equation 9:

$$
\left[\mathrm{D}_{\mathrm{i}}\right]_{\mathrm{a}, \mathrm{b}}=\left\lfloor\mathrm{X}_{\mathrm{j}}\right\rfloor_{(\mathrm{a}, \mathrm{b}) \bmod N}, \begin{gathered}
1 \leq \mathrm{a} \leq \mathrm{N} \\
1 \leq \mathrm{b} \leq \mathrm{L}
\end{gathered}
$$

is a received signal matrix $h_{i, j}(1)$ is a channel response of $\mathrm{j}$-th transmit antenna to $\mathrm{i}$-th receive antenna Equation 10:

$\mathrm{v}_{\mathrm{i}}=\left[\mathrm{v}_{\mathrm{i}}(0)_{\mathrm{i}} \mathrm{v}_{\mathrm{i}}(1), \cdots \mathrm{v}_{\mathrm{i}}(\mathrm{N}-1)\right]^{\mathrm{T}}$

is a noise vector.

To simplify the transmitter and receiver relationship, (7) can be rewritten as Equation 11 and 12:

$\mathrm{y}_{\mathrm{i}} \Gamma(\theta) \cdot \mathrm{D} \cdot \mathrm{h}_{\mathrm{i}}+\mathrm{v}_{\mathrm{i}}, \mathrm{i}=1,2, \cdots \mathrm{N}_{\mathrm{r}}$

Where:

$$
\mathrm{D}=\left[\mathrm{e}^{\frac{\mathrm{J} 2 \Pi \theta \mathrm{N}_{\mathrm{g}}}{\mathrm{N}}} \cdot \mathrm{D}_{1}, \cdots, \mathrm{e}^{\frac{\mathrm{J} 2 \Pi \theta \mathrm{N}_{\mathrm{g}}}{\mathrm{N}}} \cdot \mathrm{D}_{\mathrm{N}_{\mathrm{t}}}\right]
$$

\section{PROPOSED CFO ESTIMATOR}

\subsection{The CFO Estimator}

The likelihood function based on signal model (7) is Equation 13:

$$
\mathrm{L}\left(\theta / \mathrm{y}_{\mathrm{i}}\right)=\frac{1}{\left(\Pi \sigma^{2} \mathrm{v}_{\mathrm{i}}\right)^{\mathrm{N}}} \exp \left(\frac{-1}{\left(\sigma^{2} \mathrm{v}_{\mathrm{i}}\right)}\left|\mathrm{y}_{\mathrm{i}}-(\Gamma(\theta) \cdot \mathrm{D})\right|^{2}\right)
$$

where, $\sigma^{2}$ is the variance of the system.

\subsection{EM Algorithm Computation Steps}

\section{(A). E- Step:}

In E-step expectation is taken with respect to $y_{i}$ conditioned on $\theta$ Equation 14:

$$
F\left(\theta / \theta^{(k)}\right)=E\left\{\left[\sum_{i=1}^{N_{r}} \log L_{i}\left(\theta / y_{i}\right)\right]\right\} \mid y_{i}, \theta^{(k)}
$$

Taking real part, (14) can be further reduced as Equation 15:

$$
\mathrm{F}\left(\theta / \theta^{(\mathrm{k})}\right)=\sum_{\mathrm{i}=1}^{\mathrm{N}_{\mathrm{r}}} \operatorname{Re} \mathrm{al}\left(\mathrm{y}_{\mathrm{i}} \Gamma(\theta) \cdot \mathrm{D}\right)
$$

\section{(B). M-Step:}

M step is used for computing $(\mathrm{k}+1)^{\text {th }}$ estimate of $\theta$ which is represented as Equation 16:

$$
\theta^{(\mathrm{k}+1)}=\arg \max _{0} \mathrm{~F}\left(\theta / \theta^{(\mathrm{k})}\right)
$$

Considering real part of (16) Equation 17:

$\theta^{(\mathrm{k}+1)}=\arg \max _{0} \sum_{\mathrm{i}=1}^{\mathrm{N}_{\mathrm{r}}} \operatorname{Re} \operatorname{al}\left(\mathrm{y}_{\mathrm{i}} \Gamma(\theta) \mathrm{D}\right)$

On further expanding (17) Equation 18:

$$
\theta^{(\mathrm{k}=1)}=\arg \max _{0}\left\{\operatorname{Real}\left(\sum_{\mathrm{i}=1}^{\mathrm{N}_{\mathrm{r}}} \sum_{\mathrm{n}=0}^{\mathrm{N}-1} \mathrm{y}_{\mathrm{i}}^{*}(\mathrm{n}) \cdot \mathrm{D} \cdot \mathrm{e}^{\frac{\mathrm{j} 2 \Pi \theta \mathrm{n}}{\mathrm{N}}}\right)\right\}
$$

The exponential term of (18) is further expanded by Taylor series as Equation 19:

$$
\mathrm{e}^{\frac{\mathrm{j} 2 \Pi \theta \mathrm{n}}{\mathrm{N}}}=1+\left(\frac{\mathrm{j} 2 \Pi \theta \mathrm{n}}{\mathrm{N}}\right)-\left(\frac{2 \Pi^{2} \mathrm{n}}{\mathrm{N}^{2}}\right) \cdot \theta^{2}
$$

(19) is approximated to first two terms by considering the assumption that is very small.

By substituting (19) in (15), the likelihood function become quadratic function of $\theta^{\mathrm{k}}$. An optimum solution is obtained by taking the first order derivative and equating to zero. Hence Equation 20:

$$
\theta^{(K+1)}=\frac{-N}{2 \Pi} \cdot \frac{\sum_{i=1}^{N_{r}} \sum_{n=0}^{N-1} n \cdot \operatorname{Im}\left(y_{i}^{*}(n) \cdot D\right)}{\sum_{i=1}^{N_{r}} \sum_{n=0}^{N-1} n^{2} \cdot \operatorname{Re}\left(y_{i}^{*}(n) \cdot D\right)}
$$

\subsection{Two Stage CFO Computation} stages:

CFO estimation procedure is divided in to two

First stage-Coarse CFO estimation Second stage-Fine CFO estimation

Estimation is performed in two stages using already discussed EM iteration algorithm as above. This iteration scheme has to be performed distinctly for coarse estimation as well for fine estimation with different search interval range. 


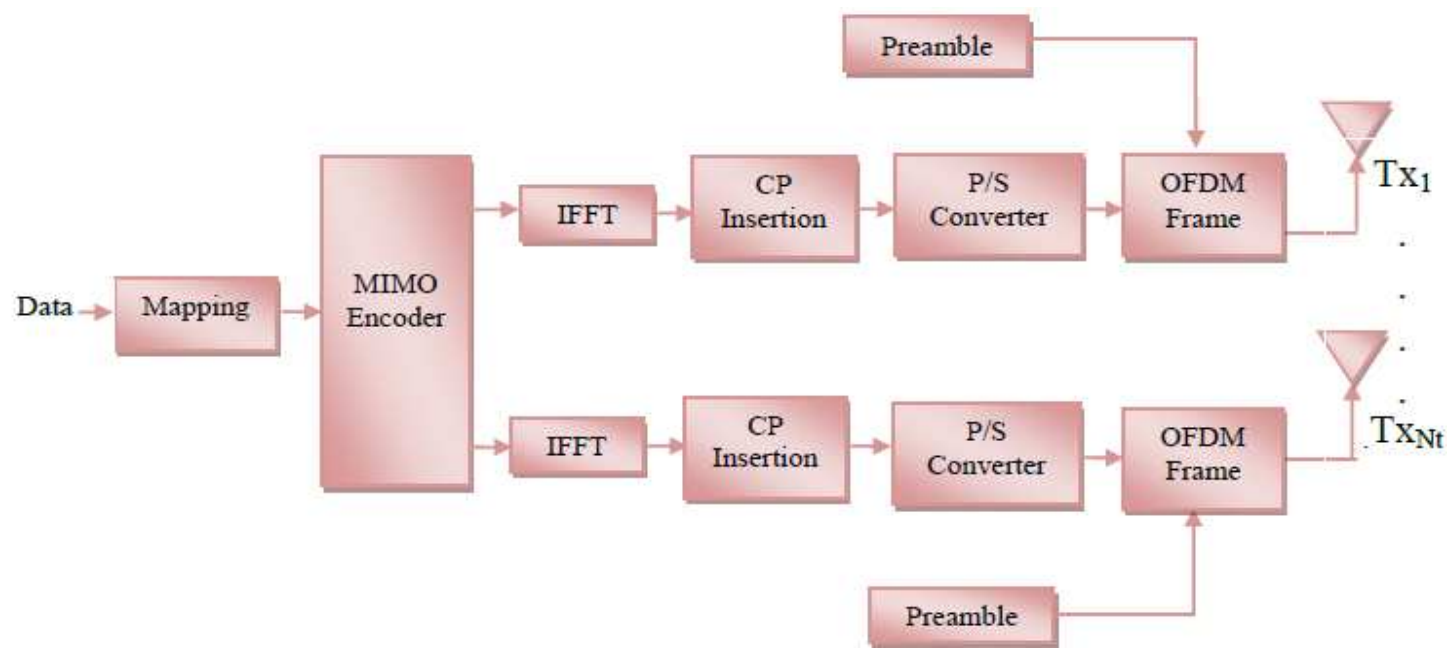

Fig. 1. MIMO-OFDM transmitter

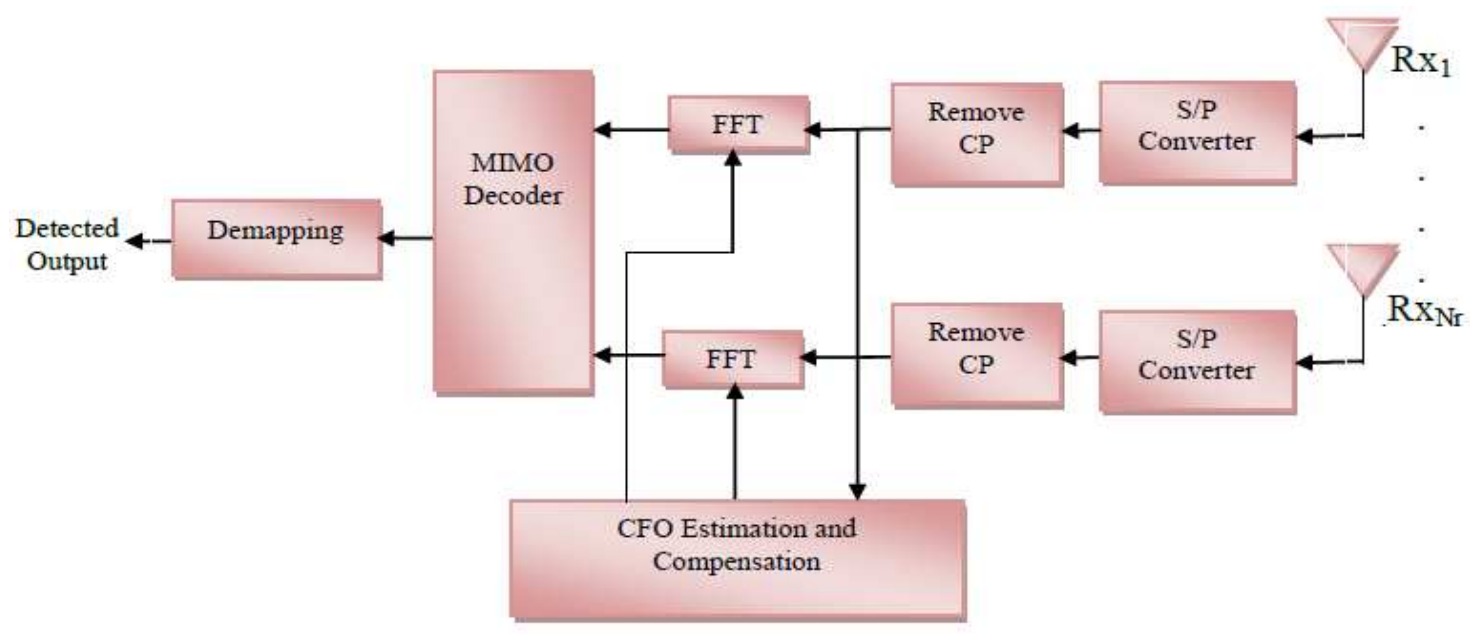

Fig. 2. MIMO-FDM receiver

\section{First Stage \\ (Coarse CFO Estimation)}

Above discussed EM based iteration scheme is applied to achieve the frequency offset in the range from -0.2 to +0.2 . This is only a coarse estimation. It is fine tuned in second stage. This stage of computation is to reduce computational complexity.

\section{Second Stage (Fine CFO Estimation)}

The obtained Carrier Frequency Offset value from the first stage is applied as initial search range in this second stage. So, search range becomes very small. Search interval can be reduced down to 0.0002. Hence with lesser number of EM algorithm based iteration, more accurate frequency offset value is achieved.

A MIMO-OFDM model as discussed in Fig. $\mathbf{1}$ and $\mathbf{2}$ is constructed and its key parameters are listed in Table $\mathbf{1}$. Computation steps procedure are described in Fig. 3.

On comparing Fig. 4 and 5 it is proved that Fig. 5 shows better BER performance than Fig. 4 , since CFO estimation is done in both coarse and fine stages (i.e., in two steps). Also, better BER performance is achieved with lesser number of iterations itself. The same is proved using data's from Table 2. 
Malarvezhi Pandy and Kumar Ramamoorthy / Journal of Computer Science 9 (11): 1526-1533, 2013

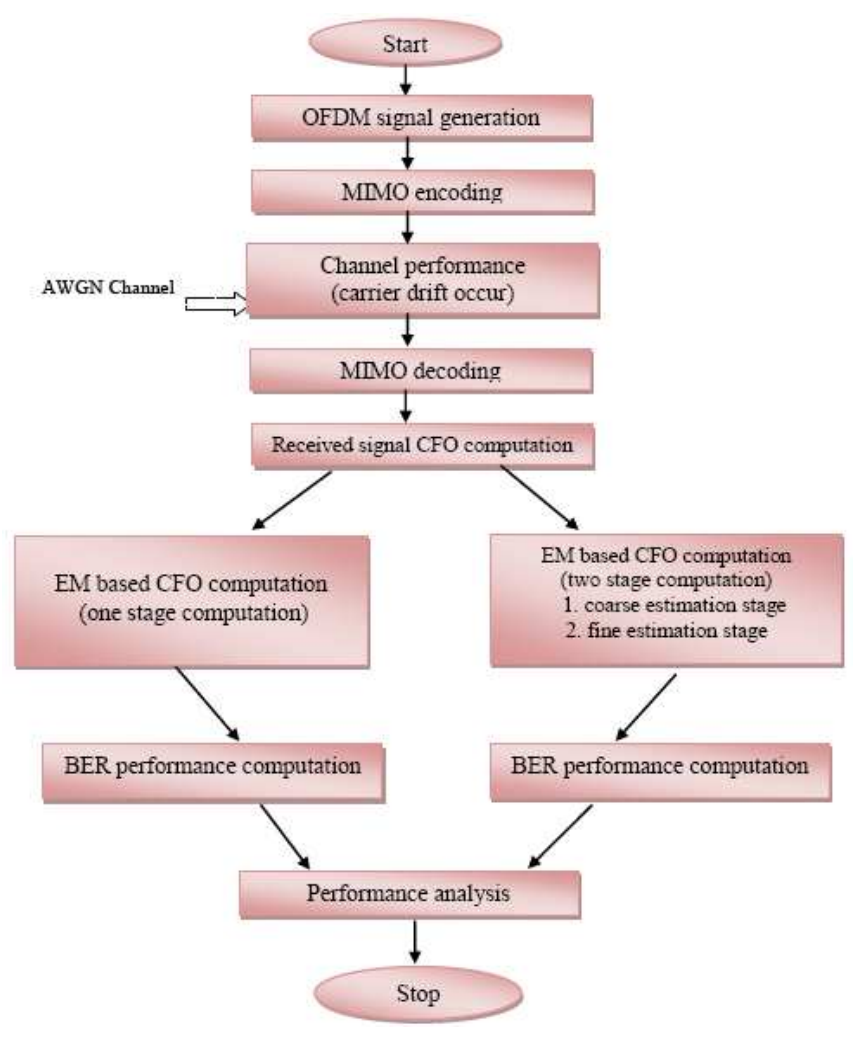

Fig. 3. Flow chart for proposed CFO computation in MIMO-OFDM system

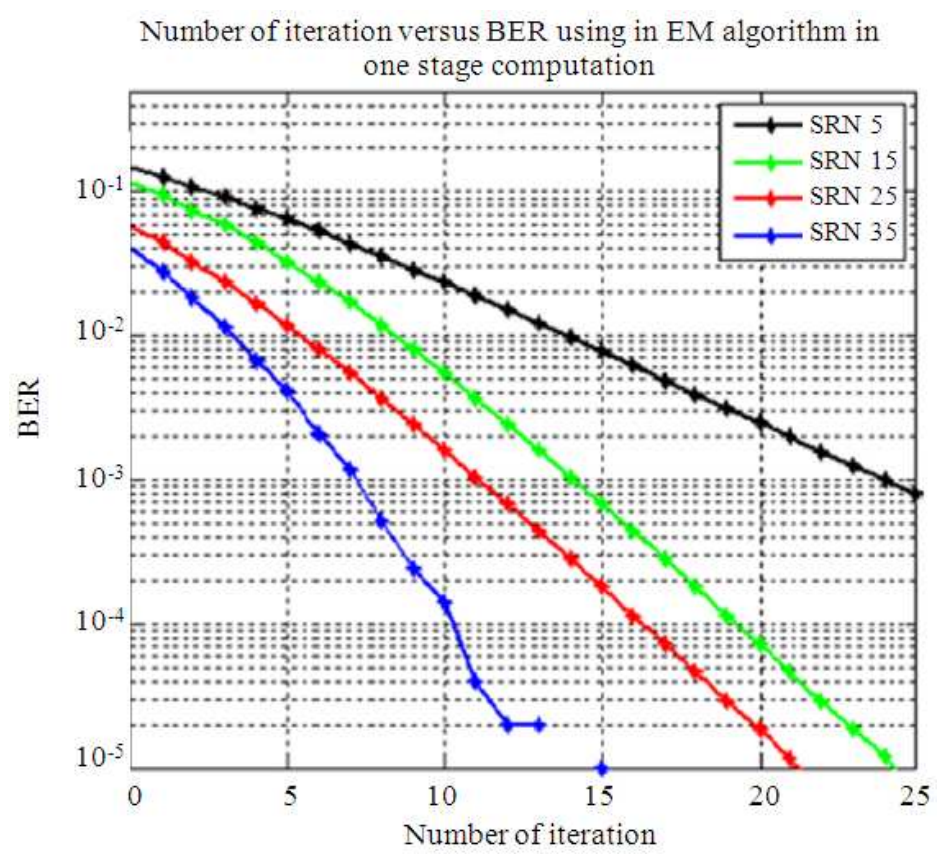

Fig. 4. Iteration versus BER for $2 \times 2$ MIMO-OFDM system for various SNR values without fine estimation (one stage computation) 
Malarvezhi Pandy and Kumar Ramamoorthy / Journal of Computer Science 9 (11): 1526-1533, 2013

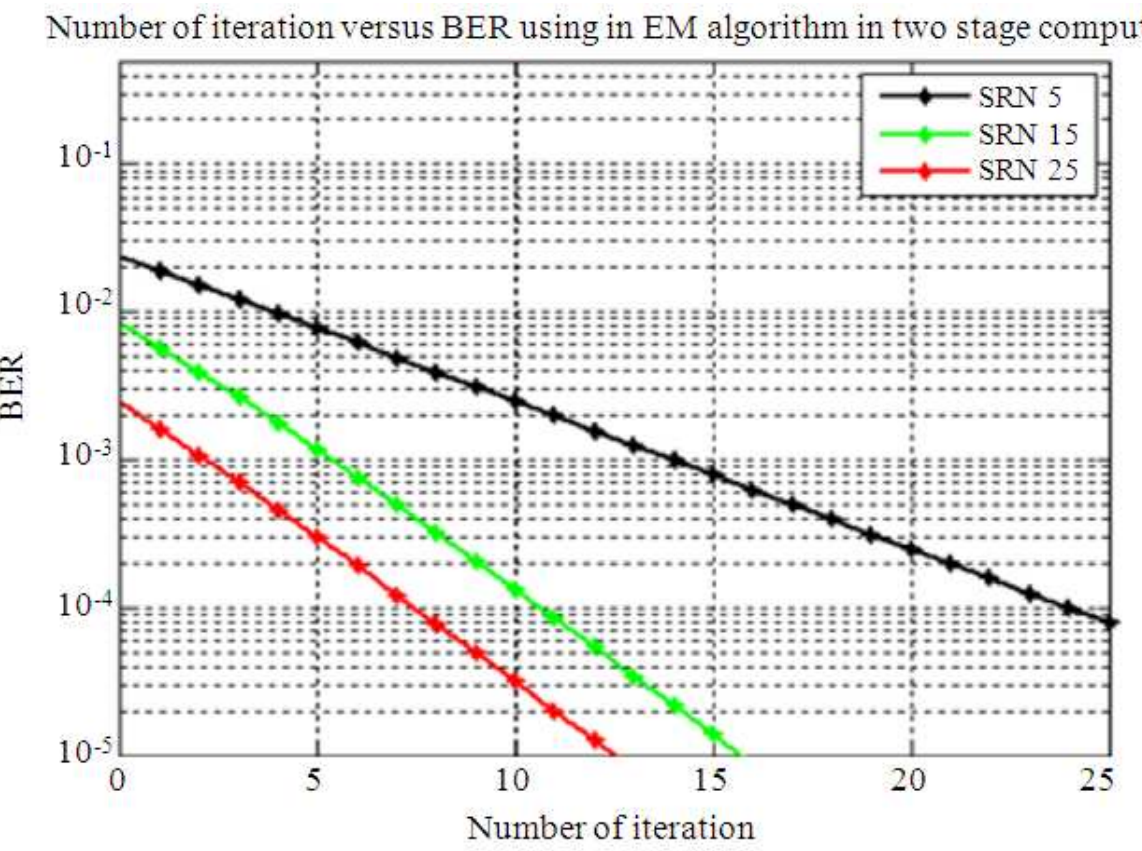

Fig. 5. Iteration versus BER for $2 \times 2$ MIMO-OFDM system for various SNR values with fine estimation (two stage computation )

SNR versus BER for a MIMO-OFDM system whit CFO randomly selected from $[-2.0,+0.2]$ and a fixed CFO 0.5

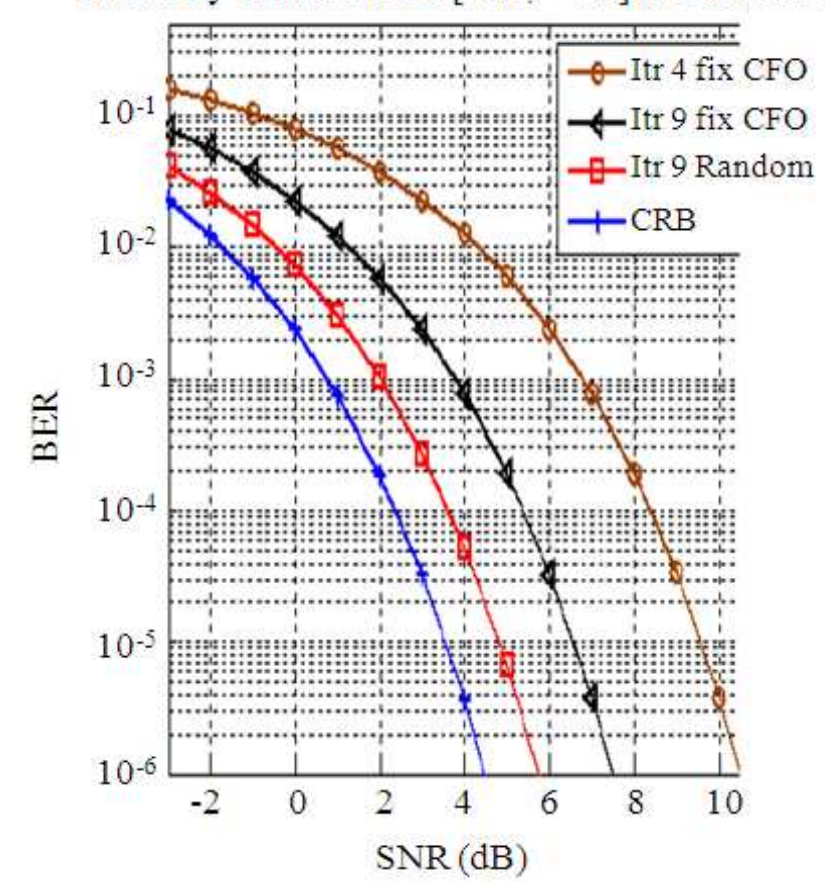

Fig. 6. SNR versus BER for $2 \times 2$ MIMO-OFDM system with random and fixed CFO values 


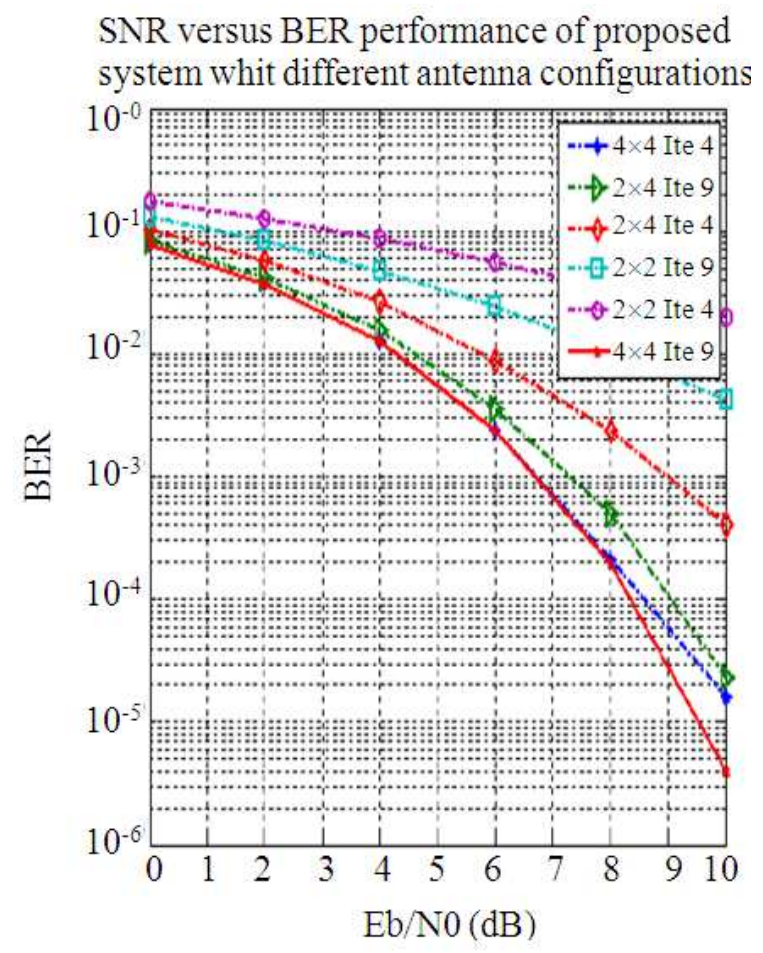

Fig 7. BER performance of proposed CFO estimator Versus SNR for different antenna configuration

Table 1. System parameters for simulations

\begin{tabular}{ll}
\hline Parameter & Value \\
\hline Subcarrier $\mathrm{N}$ & 128 \\
CP length $\mathrm{N}_{\mathrm{g}}$ & $\mathrm{N} / 16$ \\
Subcarrier symbol rate & $7.8 \mathrm{KHz}$ \\
Bandwidth & $1 \mathrm{MHz}$ \\
CFO range & {$[-0.2+0.2]$} \\
Modulation & $\mathrm{QPSK}$ \\
Symbol/frame & $8+1$ \\
Preamble & Training sequence \\
Channel & $\mathrm{TU}$ \\
Number of $\mathrm{T}_{\mathrm{x}}$ and $\mathrm{R}_{\mathrm{x}}$ & $2 \times 2,2 \times 4,4 \times 4$ \\
\hline
\end{tabular}

The illustration of simulation result in Fig. 6 is BER performance of the proposed CFO estimator for random value in the range $[-0.2+0.2]$ and fixed value with 0.5 respectively.

The BER curves for 4 iterations and 9 iterations are compared with Cramer-Rao Lower Bound (CRLB) curve. From the result it is proved that proposed CFO estimator with 9 iteration is more close to ideal value CRLB curve.

The effects of various number of antennas in MIMOOFDM systems on the CFO estimations are performed in Fig. 7. The BER performance of proposed CFO estimator for the random values $[-0.2+0.2]$ is performed for different antenna configurations like $2 \times 2,2 \times 4,4 \times 4$. From the simulation result it is proved that the performance of CFO estimation increases as the number of receiver antenna increases. Hence $4 \times 4$ with iteration 9 has improved BER performance than other $2 \times 4$ and $2 \times 2$ antenna configuration for the same 9 iterations. This gain is due to increase in receiver diversity. Table 2 gives a comparative performance analysis of MIMO-OFDM system using EM iteration algorithm for CFO computation in one stage and two stage with simulated data's.

\section{CONCLUSION}

The study proposed a novel CFO estimation and compensation in MIMO-OFDM system based on EM algorithm with iterative scheme in two stages of computation. The problem of estimating the frequency offset in MIMO-OFDM system is investigated. In the first stage called Coarse CFO estimation a simple $\mathrm{CFO}$ estimator is derived. The resulted value from the first stage is used in second stage iteration range called fine CFO estimation. Since the search range is fine tuned, a more accurate result can be achieved with lesser number of iterations itself. 
Malarvezhi Pandy and Kumar Ramamoorthy / Journal of Computer Science 9 (11): 1526-1533, 2013

Table 2. Performance analysis of EM algorithm for CFO estimation in one stage and two stage computation

\begin{tabular}{llll}
\hline Type of computation & SNR $(\mathrm{dB})$ & Number of iterations & BER \\
\hline One stage computation & 5 & 20 & $5.8 \times 10^{-3}$ \\
(Only Coarse estimation) & 15 & 20 & $0.08 \times 10^{-3}$ \\
& 25 & 20 & $0.04 \times 10^{-3}$ \\
Two stage computation & 5 & 10 & $5.4 \times 10^{-3}$ \\
(Both coarse and fine estimation & 15 & 10 & $0.12 \times 10^{-3}$ \\
& 25 & 10 & $0.05 \times 10^{-3}$ \\
\hline
\end{tabular}

Simulation results shows that the proposed CFO estimator with coarse initial estimate and fine final estimate has an excellent performance and it is more close to Cramer Rao Lower Bound (CRLB). Also, it is proved that BER performance of proposed system increases with increase in receiver diversity.

\section{REFERENCES}

Chung, Y.H. and S.M. Phoong, 2010. Joint estimation of I/Q imbalance, $\mathrm{CFO}$ and channel response for MIMO OFDM systems. IEEE Trans. Commun., 58: 1485-1492. DOI: 10.1109/TCOMM.2010.05.080627

Kaur, H. and M.L. Singh, 2012. Bit error rate evaluation of IEEE 802.16 (WiMAX) in OFDM system. Int. J. Comput. Applic., 40: 10-13. DOI: 10.5120/50147340

Mathew, B., P. George, R.V. Nathan, S. Shukkor and A.N. Lakshmi, 2013. BER comparison of DCT and FFT based OFDM systems in AWGN and RAYLEIGH fading channels with different modulation schemes. Proceedings of the Annual International Conference on Emerging Research Areas, Jun. 4-6, IEEE Xplore Press, Kanjirapally, pp: 1-4. DOI: 10.1109/AICERAICMiCR.2013.6576021

Oh, M.K., X. Ma, G.B. Giannakis and D.J. Park, 2005. Hopping pilots for estimation of frequency-offset and multiantenna channels in MIMO OFDM. IEEE Trans. Commun., 53: 162-172. DOI: 10.1109/GLOCOM.2003.1258405
Torabi, M., S. Aissa and M.R. Soleymani, 2006. MIMO-OFDM Systems with Imperfect Channel Information: Capacity, Outage and BER Performance. Proceedings of the IEEE International Conference on Communications, (ICC' 06), IEEE Xplore Press, Istanbul, pp: 5342-5347. DOI: 10.1109/ICC.2006.255510

Tseng, C.H. and C.D. Chung, 2013. Concatenated precoded OFDM for CFO effect mitigation. IEEE Trans. Veh. Technol., 62: 2618-2632. DOI: 10.1109/TVT.2013.2245929

Yue, X., X. Lei and S. Li, 2007. Pilot-aided CFO estimation in clipped OFDM system. Proceedings of the International Conference on Communications, Circuits and Systems, Jul. 11-13, IEEE Xplore Press, Kokura, pp: 231-234. DOI: 10.1109/ICCCAS.2007.6250051

Zhang, H. and X. Dai, 2010. Maximum-likelihood CFO estimation for MIMO/OFDM uplink using superimposed trainings. Proceeding of the IEEE International Conference on Wireless Communications, Networking and Information Security (WCNIS), Jun. 25-27, IEEE Explore Press, Beijing, China, pp: 247-251. DOI: 10.1109/WCINS.2010.5541723 